\title{
Specimen Preparation of Metals and Alloys for EBSD
}

\author{
George F. Vander Voort* and Philippe Pinard** \\ *Consultant - Struers Inc., 2887 N Southern Hills Drive, Wadsworth, IL \\ **Graduate Student, RWTH Aachen University, Aachen, Germany
}

Electron backscattered diffraction (EBSD) is performed with the scanning electron microscope (SEM) to provide a wide range of analytical data; e.g., crystallographic orientation studies, phase identification and grain size measurements. A diffraction pattern can be obtained in much less than a second, but pattern quality is improved by utilizing a longer scan time. Grain mapping requires development of diffraction patterns at each pixel in the field; today, collection rates of 500 or more patterns per second can be achieved which has greatly speeded up this process. The quality of the diffraction pattern, regardless of the collection time, influences the confidence in indexing the diffraction pattern and depends mainly upon the degree of removal of damage in the lattice due to specimen preparation induced damage. Our extensive experimentation has shown that mechanical specimen preparation yields excellent EBSD results for nearly all materials. It is important, of course, to know how to prepare specimens properly as an inadequate method yields inadequate results.

Based upon our EBSD experience, if mechanical preparation produces high quality polarized light images of non-cubic crystal structure elements and alloys (e.g., Sb, Be, Hf, $\alpha$-Ti, Zn, Zr), or color tint etching of cubic, or non-cubic crystal structure elements or alloys produces high-quality color images, then the surface is free of harmful residual preparation damage and EBSD patterns with high pattern quality indexes will be obtained. Automated preparation equipment is recommended, as the methods will be performed accurately and reproducibly. Manual ("hand") preparation cannot produce flatness, phase retention and damage removal as easily as automated processing and is less reproducible. We have always stressed developing the best possible mechanical preparation methods, used with a standard grinder-polisher, such as the Tegramin, before resorting to other final preparation methods, such as a brief electropolish, vibratory polish or ion-beam polish. The vast majority of metals and alloys, and other crystalline materials can be successfully prepared for EBSD with the grinder-polisher. Sectioning imparts more damage than any other step in the preparation sequence, so it is obvious that cutting damage must be minimized.

Figures 1 to 3 illustrate the depth of damage based upon band contrast data taken parallel to the affected surface at known depths for FCC Cu, BCC Fe and HCP Ti cut with a band saw and with a standard laboratory abrasive cut-off saw using the prescribed blade. The presentation will provide additional examples of the use of this technique to develop successful preparation practices. 


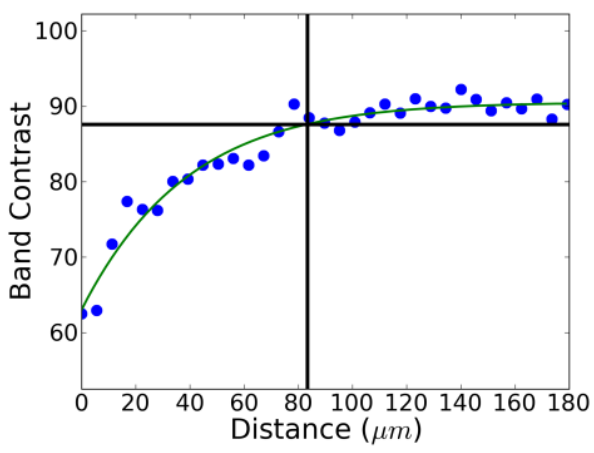

Figure 1a

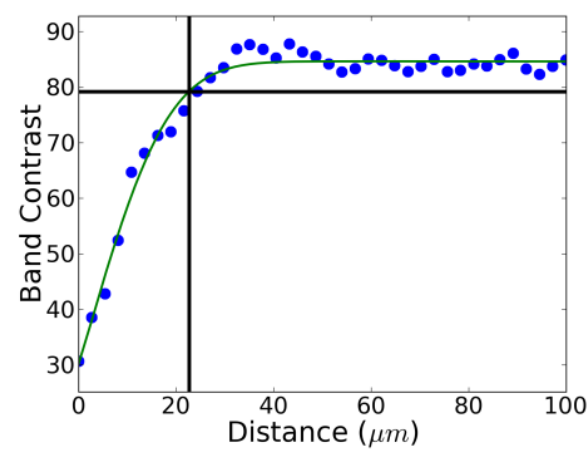

Figure 2a

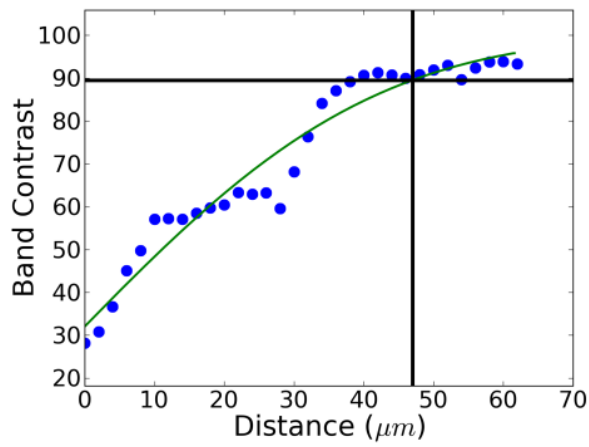

Figure 3a

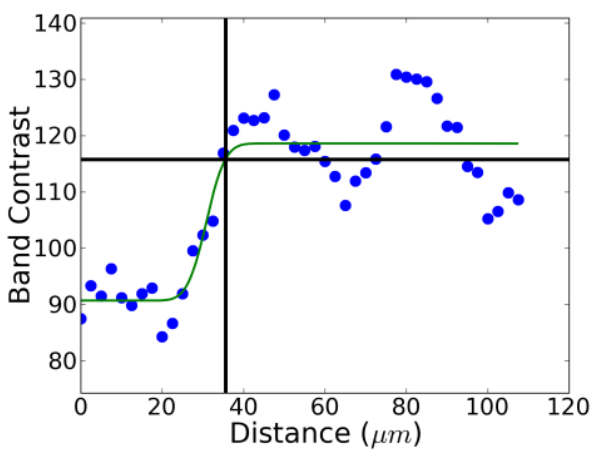

Figure $1 b$

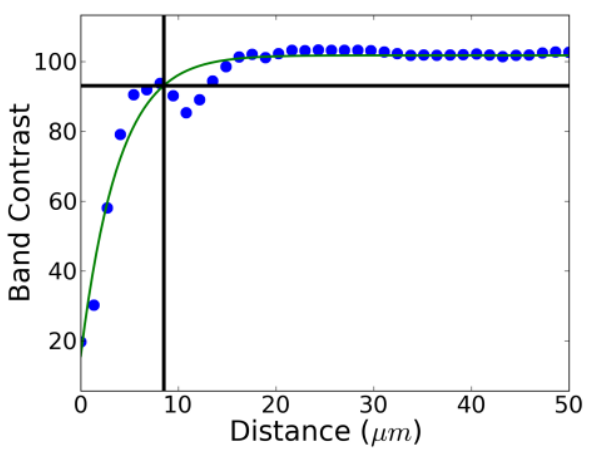

Figure $2 b$

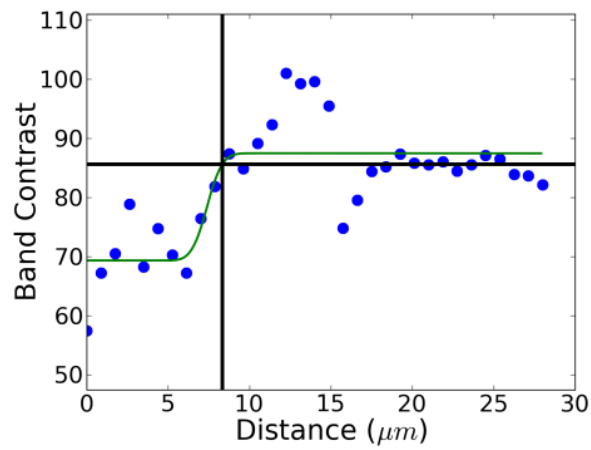

Figure $3 b$

Figures 1, 2 and 3 show band contrast as a function of depth below the surface for $\mathrm{FCC} \mathrm{Cu}, \mathrm{BCC} \mathrm{Fe}$ and HCP Ti, respectively. The (a) plots are for band sawed specimens and the (b) plots for abrasive sawing, demonstrating the substantial reduction of damage depth using an abrasive blade. 